Acta Scientifica Malaysia (ASM)

DOI : https://doi.org/10.26480/asm.01.2018.26.28

ZIBELINE INTTERNATIIONA

ISSN: 2521-5051 (Print)

ISSN: 2521-506X (online)

CODEN : ASMCCQ

\title{
A STUDY ON THE RHYTHM AND RESPIRATORY CHARACTERISTICS OF ZHUANG LANGUAGE
}

\author{
Liu Lijie, Yang Feng \\ College of Chinese Language and Culture, Jinan University, Guangzhou, China \\ *Corresponding Author Email: yangzihuai@163.com, 43578692@qq.com
}

This is an open access article distributed under the Creative Commons Attribution License, which permits unrestricted use, distribution, and reproduction in any medium, provided the original work is properly cited.

\section{ARTICLE DETAILS}

\section{Article History:}

Received 12 November 2017

Accepted 12 December 2017

Available online 1 January 2018

\section{ABSTRACT}

This research studies the features of chest and abdominal breathing in Zhuang language. Two participants were recruited to record 30 news articles of Zhuang language. The chest and abdominal breathing signals as well as speech signal were recorded simultaneously. Programs for breathing analysis have been written to extract parameters such as breathing reset amplitude, time of inhale phase, and slope of exhale phase. The results show that the times of inhale and exhale reset of abdominal breathing are earlier than chest breathing, the breathing reset is related to prosodic boundaries.

\section{KEYWORDS:}

Chest and abdominal breathing, Speech production.

\section{INTRODUCTION}

Breathing plays an important role in human speech activities. The study of breathing is an important aspect of speech production which has been discussed by many scholars. A researcher has proposed Breath-Group Theory which holds that the breath-group is a basic unit of intonation production and perception [1]. Other researcher in their studies believes that an important function of the breathing system is to produce subglottal pressure and then the sound flow [2]. It increases or decreases at the prosodic boundaries.

There also researcher has carried out researches on Chinese with signals of airflow and pressure for the first time in China [3]. Another group of researchers have studied abdominal breathing reset of Mandarin reciting of various writing styles respectively and made statistical analysis on different types of breathing reset [4,5]. In other study, some group researchers have explored mechanisms of chest and abdominal breathing $[6,7]$.

This research compares breathing features of chest and abdominal breathing in Zhuang language through analyzing simultaneous signals of chest breathing, abdominal breathing and speech sound [8]. The effects of chest breathing, and abdominal breathing and their interrelationship were researched.

\section{METHOD}

\subsection{Data Sampling}

We recorded 30 news articles of Zhuang language. All the articles are recorded in the laboratory. The major equipment for recording is 16channel Power lab PL3516. This experiment collects signals of 4 channels using Chart 5. Channel 1 is speech signal collected through microphone and console mixer; Channel 2 is phonation signal collected through Electroglottography; Channel 3 is pectoral breathing signal collected by Breathing stripe, MLT1132 sensor; Channel 4 is abdominal breathing signal also collected through Breathing stripe, MLT1132 sensor [9]. All sample rates are $44 \mathrm{kHz}$. Breathing stripe used for measuring chest breathing is fastened $10 \mathrm{~cm}$ below the armpit, while breathing stripe used for measuring abdominal breathing is fastened above the naval. Devices used in recording are: microphone, Sony ECM-44B; console mixer,
Behringer XENYX502; Electroglottography, Kay 6103; breathing strips, AD Instrument MLT1132.

\subsection{Parameter Extraction}

In order to study simultaneously the signals of speech, phonation, chest breathing, and abdominal breathing, a data analysis platform was built in Matlab. As shown in Figure 1.

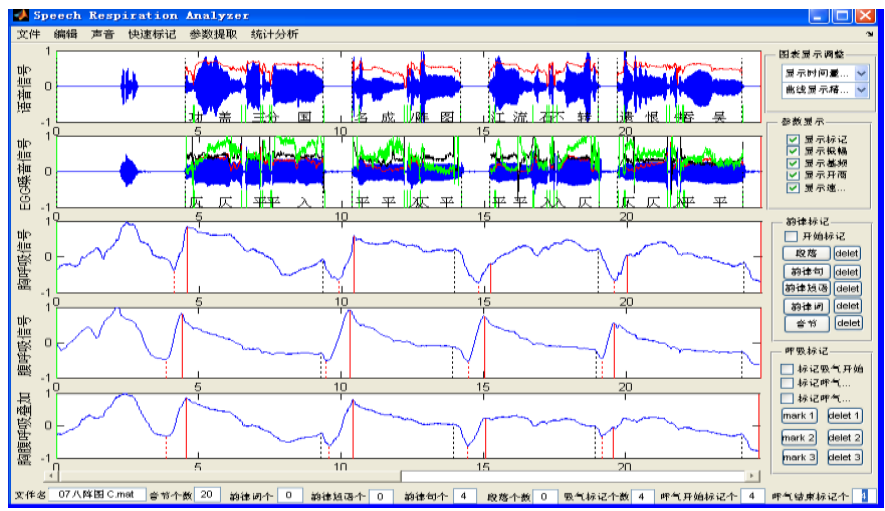

Figure 1: The interface of the data analyzing platform

In the interface of the data analyzing platform, there are five sub-windows in which five signals are displayed. The five signals, from top to bottom, are: 1) speech signal; 2) EGG signal; 3) chest breathing signal; 4) abdominal breathing; and 5) the sum of chest and abdominal breathing calculated breathing. On the right of interface, there are four function plots and in each of them, there are some functional buttons for text labeling and signal processing. 


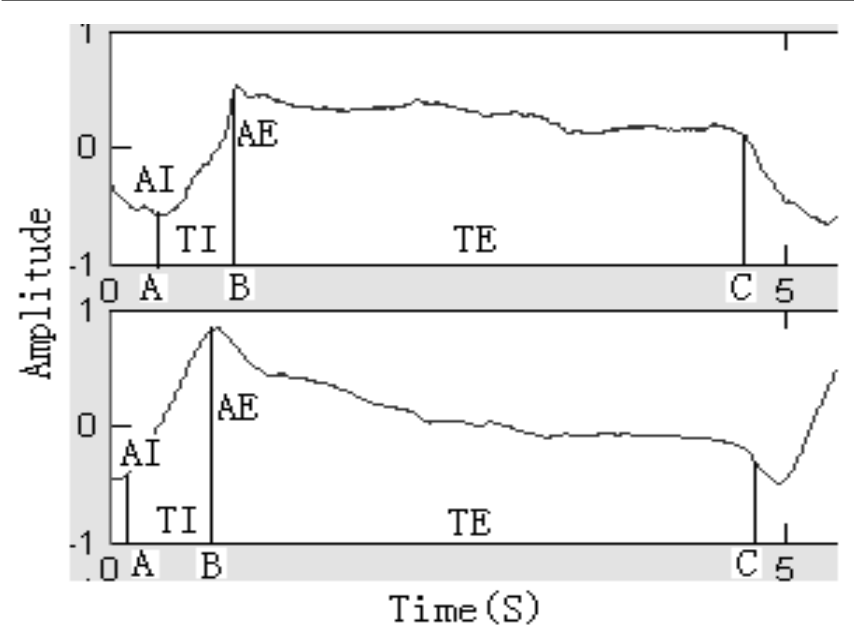

Figure 2: Definition of breathing parameters (From top to bottom: chest breathing, abdominal breathing)

The definition of breathing parameters is shown in Figure 2. The horizontal axis is time measured in seconds, while the vertical axis is the amplitude of breathing. Above is the signal of chest breathing and below the abdominal breathing [10]. Rising curve indicates inhale and falling curve exhale. $\mathrm{A}, \mathrm{B}$, and $\mathrm{C}$ are inhale onset time, breathing onset time, and exhale end time respectively. The duration of inhale $\mathrm{TI}=\mathrm{B}-\mathrm{A}$, duration of exhale $\mathrm{TE}=\mathrm{C}-\mathrm{B} . \mathrm{AI}=$ inhale reset amplitude, $\mathrm{AE}=$ exhale reset amplitude. Other parameters, like the slope of inhale period, slope of exhale period, area of inhale phase, and area of breathing phase, can be measured with those parameters mentioned above. Other parameters are calculated by the analyzing platform. The parameters are saved as. mat format to produce breathing parameters in Microsoft Excel for statistical analysis.

\section{RESULT}

\subsection{The Effect between Chest and Abdominal Breathing in Speechless Natural Breathing}

In speechless natural breathing, chest breathing is similar to abdominal breathing. The lengths of inhale phase and exhale phase are almost the same. The upper column of Figure 3 indicates chest breathing, while the column below indicates abdominal breathing. In speechless natural breathing, the amplitude of chest breathing is comparatively small, while the abdominal breathing is large. Both are obviously periodical with the inhale and exhale phase almost equal.

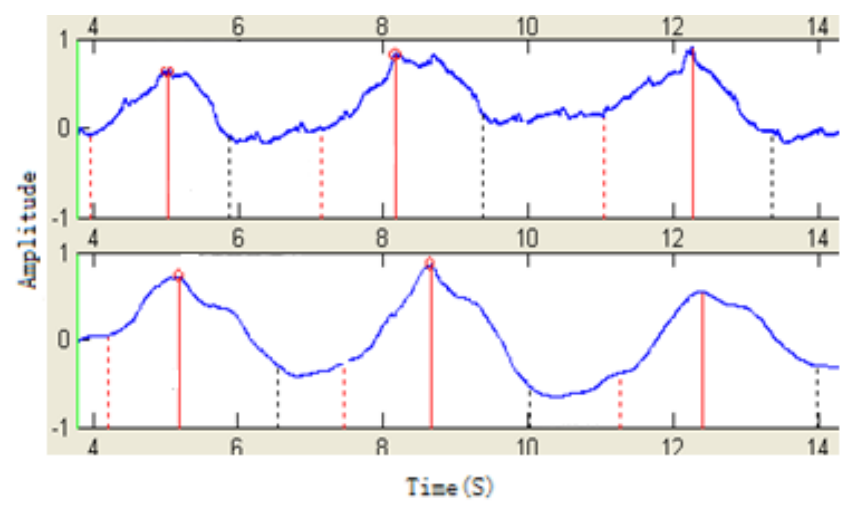

Figure 3: The chest and abdominal breathing in speechless natural breathing

\subsection{The Features of Chest and Abdominal Breathing in Zhuang Language}

There is usually a primary respiratory reset and a number of secondary and tertiary respiratory resets. As shown in figure 4, the peak of respiratory replacement can be divided into three categories: $\mathrm{A}$ is first class breath, and respiratory amplitude is 0.96 ; B is secondary breathing, and the resetting amplitude is $0.78 \mathrm{C}$ is a three-stage respiratory reset with a respiratory rate of 0.35 .

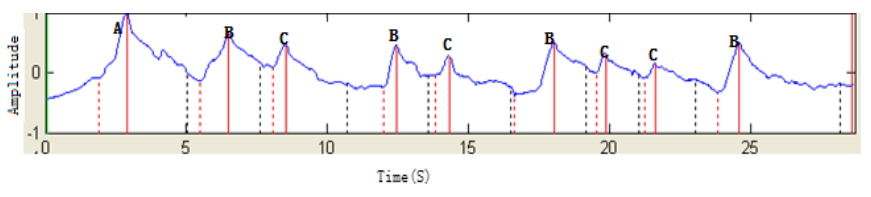

Figure 4: Abdominal breathing in Zhuang language

There is a correlation between chest and abdomen breathing and rhythm level. The first or second level of chest and abdominal breathing resets is corresponding to prosodic sentence. The three-stage breathing resets is corresponding to prosodic phrases, and the small amplitude of breath fluctuation is related to prosodic words. As shown in figure 5,

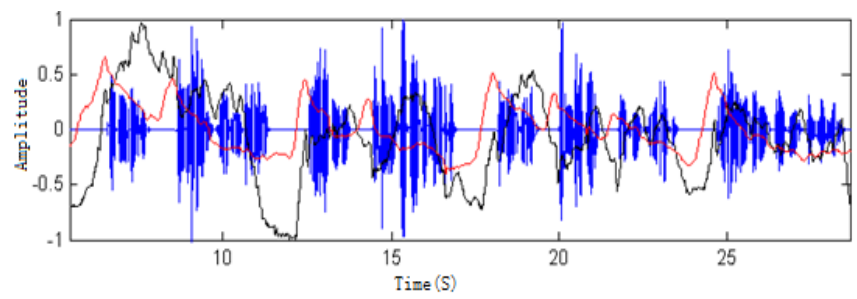

Figure 5: The chest and abdominal breathing in Zhuang language

The patterns of chest and abdominal breathing are similar. As shown in Figure 6, the onset times of sound and chest breathing overlap, that is, the chest is in the extended state when reading. The times of inhale and exhale reset of abdominal breathing are earlier than chest breathing. Sound begins after the exhale phase of abdominal breathing the curve of which falls slightly. This shows the continuous and tender contraction of abdominal muscle and diaphragm.

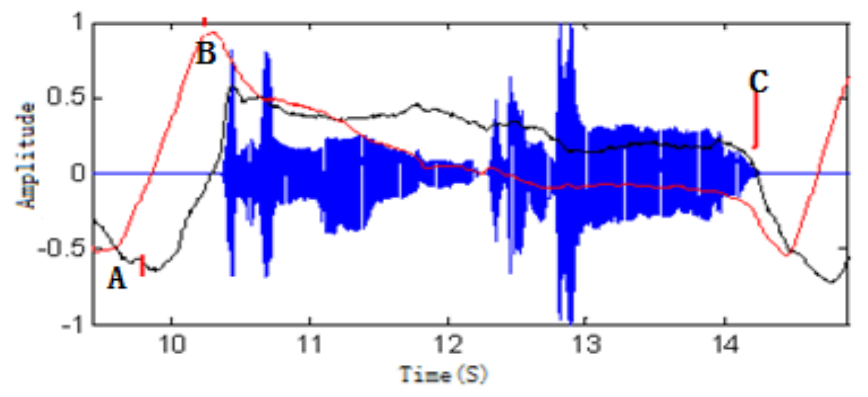

Figure 6: The relationship among chest breathing, abdominal breathing and sound

\section{CONCLUSION}

This research makes use of chest and abdominal breathing signals to study the features of chest and abdominal breathing in Zhuang language. Computer programs for breathing analysis have been written to extract parameters such as breathing reset amplitude, time of inhale phase, and slope of exhale phase. The result shows that: the times of inhale and exhale reset of abdominal breathing are earlier than chest breathing, the breathing reset is related to prosodic boundaries.

\section{ACKNOWLEDGMENT}

This study is supported by funds from National Social Sciences Funds [16ZDA211]

\section{REFERENCES}

[1] Liberman, P. 1967. Intonation, Perception, and Language. Cambridge, Massachusetts: The MIT Press.

[2] Ohala, J. J. 1990. Respiratory activity in speech. In Speech Production and Speech Modelling, ed. W. J. Hardcastle, A. Marchal, 23-53. Dordrecht: Kluwer Academic Publishers.

[3] Zongji, W., Maocan, L. 1989. A Prime of Experimental Phonetics. Beijing: Higher Education Press, 33-34.

[4] Jingjing, T., Yonghong, L., Jiangping, K. 2008. Breathing-reset when reading literature in mandarin. Journal of Tsinghua University (Science and Technology), 4, 613-620. 
[5] Shi, F., Zhang, J., Bai, X., Zhu, Z. 2012. Intonation and respiration: a preliminary analysis. Journal of Chinese Linguistics, 38 (2), 323-335.

[6] Feng, Y., Xingquan, H., Jiangping, K. 2013. The chest and belly breathing control in speech production. Journal of Tsinghua University (Science and Technology), 6, 852-855.

[7] Feng, Y. 2015. A study on the features of chest and abodominal breathing between reciting and chanting chinese poetry. Journal of Chinese Linguistics, 43 (1B), 399-410.
[8] Baken, R.J., Cavallo, S.A., Weissman, K.L. 1979. Chest wall movements prior to phonation. Journal of Speech and Hearing Research, 22 (1), 862872.

[9] Hixon, T.J., Goldman, M.D., Mead, J. 1973. Kinematics of the chest wall during speech production: Volume displacements of the rib cage, abdomen, and lung. Journal of Speech and Hearing Research, 19, 297-356.

[10] Ladefoged, P., Loeb, G. 2002. Preliminary studies on respiratory activity in speech. UCLA Working Papers in Phonetics, 101, 50-60. 